\title{
Anticoagulation in a Neurosurgical Patient with Heparin- Induced Thrombocytopenia Type II with Argatroban and Fondaparinux after Clipping of an Intracranial Aneurysm
}

\author{
Thomas Kerz $^{\mathrm{a}}$ Helmut Schinzel ${ }^{\mathrm{b}}$ \\ a Department of Neurosurgery, \\ ${ }^{\mathrm{b}}$ Medical Clinic and Policlinic II, Johannes Gutenberg University Medical School, Mainz, Germany
}

\section{Key Words}

Heparin-induced thrombocytopenia · HIT II . Neurosurgery · Argatroban · Fondaparinux

\section{Summary}

Background: Therapeutic anticoagulation in patients after a major neurosurgical procedure remains critical because of the risk of a major intracranial bleed. Novel drugs could play a beneficial role in this setting. Case Report: We describe a patient with heparin-induced thrombocytopenia type II and pulmonary embolism who was anticoagulated with argatroban and, later, with fondaparinux. No intracranial bleeding was detected when anticoagulation was performed with argatroban and, later, fondaparinux. Conclusion: Both substances could have therapeutic potential in this context.

\section{Introduction}

Heparin-induced thrombocytopenia (HIT) has an incidence of $0.1-1 \%$ with low molecular weight heparins (LMWH) and $0.1-3 \%$ with unfractioned heparins (UFH) [1]. Non-immune mediated thrombocytopenia in HIT type I (HIT I) is defined as a transient drop in platelet count within the first 5 days of heparin use, is not associated with thromboembolic events, and heparin-dependent antibodies are absent. HIT type II (HIT II) occurs typically between days 5-14, is mediated by antibodies, and causes platelet activation, intravascular consumption of platelets, and increased thrombin generation. Characteristic clinical signs are a drop in platelet count of $>50 \%$, associated with new thromboembolic complications during heparin therapy in up to $70 \%$ of the cases. We describe a case of HIT II in a neurosurgical patient which was successfully managed with the initial use of argatroban and, later, fondaparinux.

\section{Case Report}

A 62-year-old patient was admitted with a subarachnoidal hemorrhage from the basilar artery and hydrocephalus. An external ventricular drainage was placed and the aneurysm successfully clipped. Routine prophylaxis for venous thromboembolism was carried out by the administration of LMWH (dalteparin 5,000 IU/day) subcutaneously since postoperative day 1 . There was no history of former heparin exposure. Sedation was stopped but the patient remained comatose due to diffuse brain stem infarction. She was a long-time COPD (Chronic Obstructive Pulmonary Disease) patient and showed new infiltrations on the chest X-ray at day 4, and tracheal secretions were positive for Staphylococcus aureus. The fall of platelet counts during the first days was attributed to a septic constellation. She was placed on appropriate antibiotic therapy and C-reactive protein values decreased, but on day 6 she suddenly needed increasing doses of catecholamines. Chest CT on this day showed extensive pulmonary embolism in both upper and lower lung lobes and the right middle lobe. Thrombosis of the right femoral superficial vein was also detected. Due to major right ventricular dysfunction, a surgical embolectomy was carried out. During and after the intervention, therapeutic anticoagulation was achieved through intravenous UFH administration, monitored by the activated partial thromboplastin time (aPTT) (1.5-3 times baseline). Dalteparin was discontinued.

Platelet counts on the day of admission were $370 / \mathrm{nl}$ and $194 / \mathrm{nl}$ on the day of pulmonary embolism. Then they fell to $70 / \mathrm{nl}$ on day 11 when HIT II was suspected (fig. 1). Heparin was replaced by argatroban, and platelet counts were back to $159 / \mathrm{nl}$ on day 15 . A PF4/heparin ELISA test on day 11 was negative. Unfortunately, we had no access to a functional platelet assay. A thrombophilia study (factor V Leiden mutation, antiphospholipid antibodies, prothrombin mutation G20210A, protein C and $\mathrm{S}$, antithrombin, lupus anticoagulant) also remained negative. Argatroban was given by continuous infusion for 7 days when it was replaced by fondaparinux $2.5 \mathrm{mg} 2$ times per day. A retrievable inferior vena cava filter (rIVC) was implanted on the same day. Cranial CT scans showed no signs of intracranial bleeding during the anticoagulation therapy.

\section{KARGER}

Fax +497614520714

Information@Karger.de

www.karger.com

\section{(c) 2009 S. Karger GmbH, Freibur}

Dr. Thomas Kerz

Neurochirurgische Klinik und Poliklinik

Universitätsklinikum Johannes-Gutenberg-Universität

Langenbeckstraße 1, 55131 Mainz, Deutschland

Tel. +49 6131 172006, Fax -176634

kerz@uni-mainz.de 


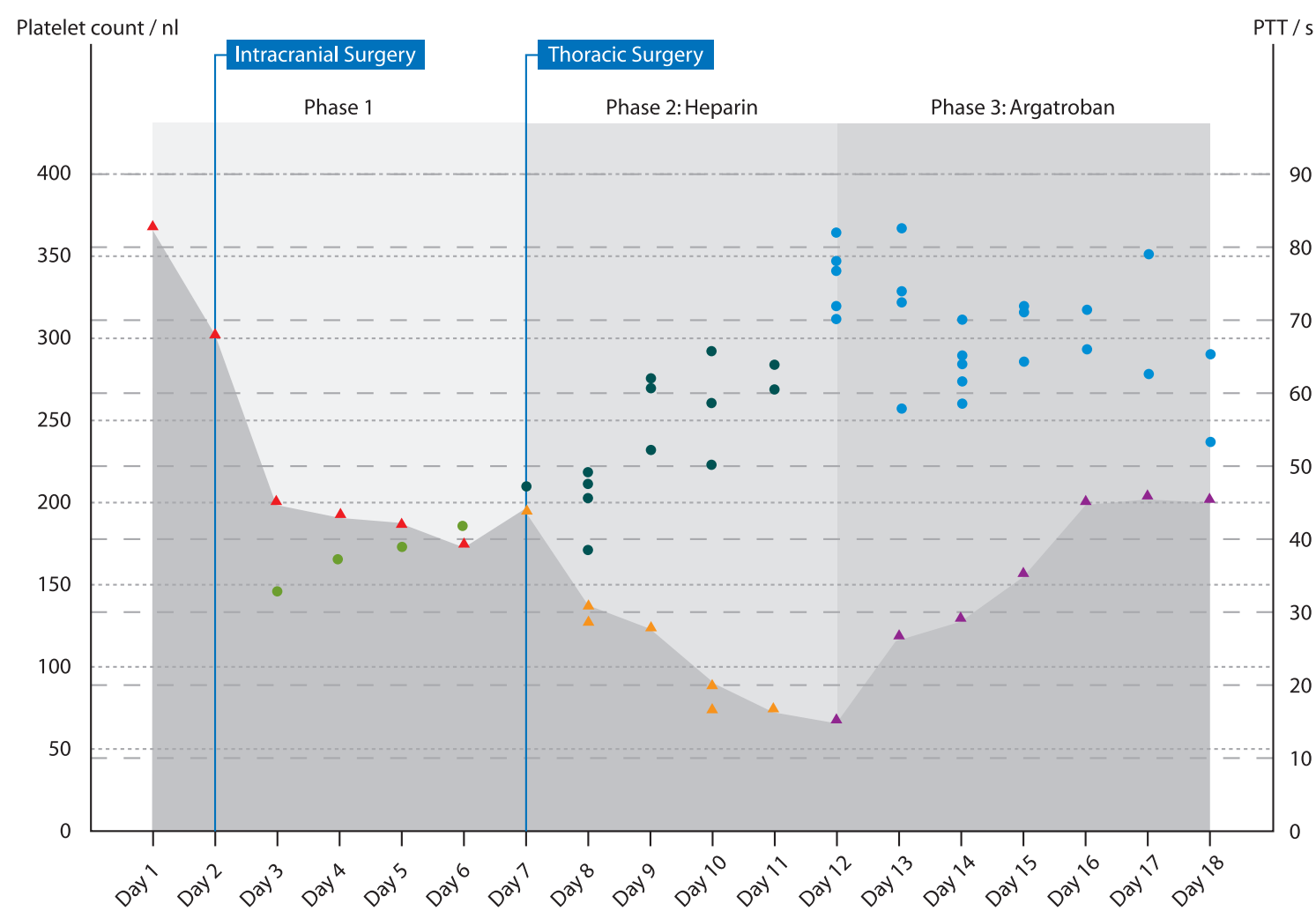

Fig. 1. aPTT and platelet count in the first phase, under heparin and under argatroban.
- PTT under administration of heparin
- PTT under administration of argatroban

- Platelet count

$\Delta$ Platelet count under administration of heparin

- Platelet count under administration of argatroban

\section{Discussion}

We report a case of argatroban and fondaparinux use in a neurosurgical patient 6 days after clipping of an intracranial aneurysm.

The diagnosis of HIT II is not straightforward and often can only be made on clinical grounds as was the case in this patient. We suspected HIT II because of the constant fall of the platelet counts and a newly developed deep vein thrombosis and pulmonary embolism 6 days after initiation of therapy with UFH. A negative result of the PF4/heparin ELISA test as in our patient does not necessarily rule out HIT II. Though one study found a negative PF4/heparin ELISA test to carry a $100 \%$ negative predictive value [2], others have reported that serotonin release assays were positive in a small minority of patients with a clinical suspicion of HIT II while the ELISA test was negative [3]. Repeated ELISA testing then often leads to a positive result but was not performed in our case. However, our patient scored 8 points on the 4 Ts (thrombocytopenia, timing of platelet count fall, thrombosis, other causes of thrombocytopenia) scale and therefore had a high probability for HIT II [4]. Other reasons for thrombocytopenia had been excluded or seemed less likely: Platelet count decrease was too slow for HIT I. Pulmonary embolism could have been developed without the patient suffering from HIT II, but platelet counts decreased already before the embolic event. Ongoing sepsis was unlikely as the pneumonia was improving with the antibiotic therapy, and there were no laboratory signs of disseminated intravascular coagulation. Drug-induced thrombocytopenia was improbable as this generally develops only after 7-14 days following the introduction of a new drug. The patient had not had any transfusion prior to the pulmonary embolectomy, thus excluding posttransfusion purpura. Most importantly, platelet counts returned to normal several days after cessation of heparin therapy which confirms the diagnosis of HIT II.

This patient presented a therapeutic dilemma as she had undergone a major neurosurgical operation but needed therapeutic anticoagulation because of her pulmonary embolism and deep vein thrombosis. Furthermore, heparin administration had probably led to a HIT II syndrome, thereby necessitating alternative anticoagulation strategies. We decided to use argatroban because it is easily titrable and its effect wears off 2-4 h after cessation of therapy [5]. Furthermore, argatroban has been utilized in patients with acute ischemic stroke without a higher incidence of symptomatic intracranial hemorrhage as compared to control patients [6]. When the acute phase was over and the platelet count was $>100 / \mathrm{nl}$, we switched to fondaparinux which can be given subcutaneously, thereby obviating intravenous therapy. The implantation of a rIVC could only take place some days after the 
embolic event as the filter was not readily available. Until our patient was treated, fondaparinux had not been shown to have a cross-sensitivity in HIT II syndrome, so we did not see any obstacle to use it in our patient [7]. However, a case report from 2007 associates HIT II with the use of fondaparinux [8]. To conclude, there have been no reports up to date on the use of either argatroban or fondaparinux in such a setting. Although large studies are missing, both substanc- es could have therapeutic potential in this context. Yet, the role of fondaparinux needs further elucidation in view of the recent case report.

\section{Disclosure}

The authors declared no conflict of interest.

\section{References}

1 Girolami B, Girolami A: Heparin-induced thrombocytopenia: a review. Semin Thromb Hemost 2006;32: 803-809.

2 Verma AK, Levine M, Shalansky SJ, Carter CJ, Kelton JG: Frequency of heparin-induced throm bocytopenia in critical care patients. Pharmacotherapy 2003;23:745-753.

3 Shelat SG, Tomaski A, Pollak ES: Serologic results in $>1000$ patients with suspected heparin-induced thrombocytopenia. Clin Appl Thromb Hemost 2008;14:410-414.
4 Warkentin TE, Heddle NM: Laboratory diagnosis of immune heparin-induced thrombocytopenia. Curr Hematol Rep 2003;2:148-157.

5 Escolar G, Bozzo J, Maragall S: Argatroban: a direct thrombin inhibitor with reliable and predictable anticoagulant actions. Drugs Today (Barc) 2006;42: 223-236.

6 LaMonte MP, Nash ML, Wang DZ, Woolfenden AR, Schultz J, Hursting MJ, Brown PM; ARGIS1 Investigators: Argatroban anticoagulation in patients with acute ischemic stroke (ARGIS-1): a randomized, placebo-controlled safety study. Stroke 2004;35:1677-1682.
7 Efird LE, Kockler DR: Fondaparinux for thromboembolic treatment and prophylaxis of heparininduced thrombocytopenia. Ann Pharmacother 2006;40:1383-1387.

8 Warkentin T, Maurer B, Aster R: Heparin-induced thrombocytopenia associated with fondaparinux. $\mathrm{N}$ Engl J Med 2007;356:2653-2655. 